\title{
The Adoption of Semantic Annotations of Products in Web Shops
}

\author{
Goran Matošević
}

\begin{abstract}
In this paper we explore the use of semantic web technologies to mark up data in web pages in the most popular web shops today. Semantic annotations i.e. insertion of structural data within existing HTML document is done for the purpose of enabling aggregating data from various sources that may use different web applications, portals and web search engines. While there are a few studies about the global use of these technologies, there is no any existing detailed research in the field of e-commerce. Also the emergence of new standards (Microdata) and ontologies (GoodRelations and Schema.org) in the field of e-commerce requires more detailed insight into the actual use of the above mentioned. Results of this study show the current situation in this field, and can serve to web shops as a guide for the use of semantic annotation but also to scientists for further research.
\end{abstract}

Index Terms-Semantic web, semantic annotations, e-commerce, product search, microdata, microformats.

\section{INTRODUCTION}

Product search on the web is becoming increasingly important due to the constant increase of online shopping. Also the number of Internet stores and products that are offered in them has become larger, and for users in search of the product is more difficult to decide where and which model of product to buy [1]. This situation creates several problems. There is a variety of identical products described in different ways in different Internet stores. Furthermore, the products have been described in different languages. This leads to difficulties in the aggregation of data about the products which various portals and search engines could use. Currently the user seeking a particular product on the web using Internet search engine by entering keywords in the search field, should examine i.e. visit several websites from the search results in order to obtain the information that is requested, or choose a web shop where he wants to make a purchase considering the cost, shipping terms, and user comments. Search is done using keywords and search results are pages that contain those keywords. There is no possibility of parameter's type of searching i.e. "The blue Nike sneakers that cost between $\$ 50$ and \$ 100 (USD)." This type of search uses only a few specialized Internet stores such as Shopping.com and Shopzilla.com, but these search engines only support basic product properties [2]. In order for search engines like Google, Yahoo and Bing could return results of parameter's inquiries over all indexed Internet stores, they need a system of

Manuscript received September 30, 2013; revised January 22, 2014

G. Matošević is with the Faculty of Economics and Tourism, University of Pula, Croatia (e-mail: gmatosev@unipu.hr). ontology and semantic annotation of products in HTML. Also, if we want to build a portal that aggregates data from multiple Internet stores, it is necessary to find a solution that will easily be able to withdraw products, compare them and aggregate. Solution is the semantic web and tagging data (semantic annotation) within HTML using a predefined vocabulary.

\section{Semantic Web AND AnNotation OF Content}

Semantic web is a new generation of web that adds meaning to data on the web. Today's web is understandable to humans, but not understandable to machines. Semantic web extends the current one by making informations meaningfully for humans and computers, enabling the description of contents in machine-readable form [3], [4]. Semantic web makes it possible to objects from the real world to describe themselves on web pages so that they are understandable also to the machinery. These objects can be people, companies, products, etc. It is also possible to define relationships between objects. If two or more web pages in the same way denote these objects, then the integration of data is very simple too. Different technologies and pre-defined vocabularies (ontologies) can be used for semantic annotation of content. Specific domains can require specific ontologies. In this way, the semantic search engines can combine data that represent the same object from the real world from many different sources and display aggregated results.

Semantic annotation implies insertion i.e. adding of some tags and attributes in the existing HTML code. Currently there are three ways i.e. standards by which we can do this: RDFa, microformats and microdata [5]. Microdata and microformats are very easy to implement, but unlike RDFa, they provide fewer opportunities. Microdata are the latest standard recommended by the largest search engines Google, Yahoo and Bing. They developed (2011) unique vocabulary to indicate the most common objects such as people, products, events, reviews, etc. These are general ontology that is available on schema.org. Microformat has its own ontology and the method of inserting the HTML code, while RDFa recommendation of the World Wide Web Consortium (W3C) is slightly more complicated to implement.

\section{A. Microformats}

Microformats is approach of labeling i.e. inserting structural data into the existing XHTML code using the existing HTML attribute "class" that has been used to define Cascading style sheets (CSS) classes [6]. Microformats specification includes several predefined elements that describe different objects, such as "hCalendar" for events, "hCard" for persons or organizations, "XFN" for the relations 
between people, etc. ${ }^{1}$ In addition to adopted specification there are so called "draft specification" which consists of elements that are currently undergoing a process of standardization and should be used with caution since their syntax still can change. Among them are the elements of interest for e-commerce: "hProduct", "hReview" and "hReview-aggregate". They serve to describe the products and their review and rating. The following example shows how microformats are used to annotate a product with review:

$<$ div class="hproduct" $>$

$<$ span class="'fn" $>$ Apple iPad $2</$ span $>$

$<$ span class="description" $>$ Things come alive on the stunning 9.7-inch widescreen LED Multi-Touch display of the Apple iPad 2. With WiFi support, this 16GB Apple iPad ensures you stay connected to your world all the time.

$</$ span $>$

Sale price: $<$ span class $=$ "price" $>\$ 300.00</$ span $>$

Average rating:

$<$ span class="hreview-aggregate" $>$

$<$ span class $=$ "rating" $><$ span class $=$ "average" $>4.9</$ span $>$, based on $<$ span class $=$ "count" $>99</$ span $></$ span $>$ reviews $</$ span $>$

$</$ div $>$

\section{B. Microdata}

Microdata are another way in which we can add semantic markups in XHTML. It is a standard that emerged from Schema.org initiative that in 2011 launched the largest Internet search engines Google, Yahoo, Bing and Yandex in order to show in their search results besides the usual titles and descriptions of website some additional structural information (called "rich snipets"), and thereby improve the user experience [7]. Syntax of inserting microdata involves the use of three main attributes: itemscope, itemtype and itemprop that can be loaded into any existing HTML tag.

To define the structural data is used Schema.org vocabulary that contains several classes organized in a hierarchy ${ }^{2}$ and in the code are referred to by the full URL. Microdata unlike Microformats include wider and more advanced vocabulary and allow the use of other vocabularies by simple URL referencing. Existing class Schema.org can be easily expanded, and their hierarchical structure allows for a detailed description of the data. For e-commerce Schema.org has class "Product" and can be used in conjunction with the "Offer", "Brand", "Review", "AggregateRating" etc. The following example shows the same product from previous example labeled with Microdata and Schema.org vocabulary:

$<$ div itemscope itemtype="http://schema.org/Product" $>$

$<$ span itemprop=""name" $>$ Apple iPad $2</$ span $>$

$<$ span itemprop="description" $>$ Things come alive on the stunning 9.7-inch widescreen LED Multi-Touch display of the Apple iPad 2. With WiFi support, this 16GB Apple iPad ensures you stay connected to your world all the time.

$</$ span $>$

$<$ span itemprop="offers" itemscope

\footnotetext{
${ }^{1} \mathrm{http}: / /$ microformats.org/wiki/Main_Page\#Specifications

${ }^{2}$ http://www.schema.org/docs/full.html
}

itemtype="http://schema.org/Offer">

Sale price: $\$<$ span itemprop="price" $>300.00<$ span $>$

$<$ meta itemprop=" priceCurrency" content="USD" $/>$

$</$ span $>$

$<$ span itemprop="aggregateRating" itemscope

itemtype="http://schema.org/AggregateRating">

Average rating 〈span itemprop="ratingValue" >

$4.9</$ span $>$,

based on $<$ span itemprop="reviewCount" $>99</$ span $>$ reviews

$</$ span $>$

$</$ div $>$

\section{C. $R D F a$}

Resource Description Framework in attributes (RDFa) is a format that allows the insertion of RDF triples in the HTML document [8]. Syntax involves the use of URL to reference the vocabulary and elements of triplets. Facebook's Open Graph Protocol is the biggest supporter of RDFa. By using RDFa we can use any vocabulary. Our third example shows the labeling of products with GoodRelations ontology in RDFa:

$<\operatorname{div}$

xmlns:gr="http://purl.org/goodrelations/v1\#"

xmlns:xsd="http://www.w3.org/2001/XMLSchema\#"

xmlns:v="http://rdf.data-vocabulary.org/\#"

typeof="gr:Offering" about="\#product_data" >

<span property="gr:name">Apple iPad 2</span>

$<$ span property="gr:description"> Things come alive on the stunning 9.7-inch widescreen LED Multi-Touch display of the Apple iPad 2. With WiFi support, this 16GB Apple iPad ensures you stay connected to your world all the time. $</$ span $>$

<span rel="gr:hasPriceSpecification">

<span typeof="gr:UnitPriceSpecification">

Sale price: $\$<$ span property="gr:hasCurrencyValue"

datatype $=$ "xsd:float" $>300.00</$ span $>$

$<$ span property="gr:hasCurrency" content="USD"

datatype="xsd:string" $>\langle/$ span $>$

$</$ span $>$

$</$ span $>$

$\langle$ span rel="v:hasReview">

$<$ span typeof="v:Review-aggregate"

about="\#review_data" $>$

Average rating: $\langle$ span property="v:average"

datatype $=$ xsd:string" $>4.9</$ span $>$,

based on $<$ span property="v:count"

datatype="xsd:string" $>99</$ span $>$ reviews

$</$ span $>$

$</$ span $>$

$</$ div $>$

\section{E-Commerce Ontologies}

Ontologies are used for modeling knowledge from a specific domain. They allow you to add semantic meaning of the existing concepts and to define mutual relations [4]. In the area of products and services were developed general ontologies Schema.org/Product, eClassOWL and unspscOWL. Hepp in his work [9] exposes problem of lack of detailed ontology for describing products and services, and 
suggests his ontology that he calls "GoodRelations" which today is regarded as the most complete ontology for e-commerce. Schema.org/Product vocabulary already contains some elements from GoodRelations Ontology and their recommendation is that GoodRelations should be used in the annotation of products.

\section{RELATED WORK}

Reference [5] analyzed and compared the approaches to semantic tagging and publishing of machine-readable data on the web. They divide the approaches as "inline" and "parallel". Inline approach refers to the insertion of extra tags into existing HTML files in order to add semantic meaning to them and thus also allow to machines easier reading of data. The authors cite three approaches i.e. technologies by which this is possible: RDFa, Microdata and Microformats. A parallel approach implies the existence of parallel files that contain only data. In this approach belongs Linked data as the only approach that now allows publishing of large sets of data on the Web using RDF standards.

Reference [10] investigated the use of inline semantic tagging by processing publicly available data set of downloaded websites from the projects Common $\mathrm{Crawl}^{3}$ and Web Data Commons. ${ }^{4}$ Common Crawl is a non-profit organization that collects data i.e. websites using computer programs called Spiders, in a manner similar to that used today by modern Internet search engines. The collected data are published on its web site, and are publicly available for everyone to download. Until today there are two such sets of data, one from 2009/2010 and the second from February 2012 , which together contain 4.5 billion of web pages. Web commons project uses mentioned sets of data in order to pull out from them structural data, and then publish them in the RDF format. Reference [10] showed and compared the most common formats that are used in two sets of data to show change over time and trends of the use of technology for inline semantic labeling. This study included all three technologies, $\mathrm{RDFa}$, Microformats and Microdata, and has shown a significant increase in the use of RDFa and Microdata, and the stagnation in the use of Microformats (by comparing the years 2009/2010 and 2012). The authors also show the use of vocabulary i.e. ontology in the specified formats.

Similar research to [10] conducted [11] by using data from the Internet's search engine Bing indexed in January 2012. The same authors conducted a similar research using data of search engine Yahoo. Their research differs from the previous one, except in the data source and methods of data extraction and display of the results in the form of triplets. Thus, for example, they note that Facebook.com has the most triples if we only look at RDFa, MySpace.com in microdata technology and Yahoo.com in microformats. Besides the number of triplets, research shows the number of pages that contain certain formats separating at the same time $\mathrm{RDFa}$ from OGP's ${ }^{5}$. Also it describes the use of ontology for each format. Their analysis showed that $30 \%$ of websites contain

\footnotetext{
${ }^{3}$ http://commoncrawl.org

${ }^{4} \mathrm{http}: / /$ webdatacommons.org

${ }^{5}$ Facebook Open Graph Protocol, http://ogp.me
}

some form of metadata.

Our research differ from previously mentioned in focus on e-commerce web sites i.e. Internet commerce and the data source itself - we will take into account the top of 100 most popular (according to the traffic) Internet shops (stores). Research will show the current situation in this industry.

Authors of [2] in their work have presented a platform for product search supported by semantic web. This work is a direct example of the use of semantic tagging products in Internet stores. Their solution is available on www.XploreProducts.com where visitors can try out the product search. Platform as a source uses its own database that is constantly updated by pinging web shops that use RDFa for semantic labeling. Limitations of this study and the proposed algorithms can be seen in just one technology (RDFa) and ontology (schema.org) that were used. The authors propose further research using eClassOWL and GoodRelations ontology. This article provides a motivation for our research that would show how much potential currently have such systems. To what extent is semantic tagging today present in web stores to make such a system become a reality?

\section{THE RESEARCH METHODOLOGY}

To determine the degree of use of semantic annotations in the most popular web shops we used a list of the top $100 \mathrm{Web}$ stores ranked by Alexa.com in the category „Shopping““ ${ }^{6}$ By simple inspection of the HTML website code of product details page we can determine the usage of semantic annotations and vocabularies. For each web shop from the list, we chose a random product and investigate the product page by inserted the URL into the „Google structured data testing tool ${ }^{67}$ which detects structural information on the website. The data obtained are sorted by technology, vocabulary and namespaces.

\section{THE RESUlTS AND ANALYSIS}

The research results presented in Table I and Table II show the use of certain formats and namespaces or types of entities. The most commonly used is Open Graph Protocol (OGP), which uses 66 web shops, and usually in the most rudimentary form, using only the basic elements. OGP cannot be considered the right format for labeling of products because it is stated in meta data in the header of the page. However, we have included it in our research because it still includes some metadata that serves social networks to display shared link. Purpose of the product description contained in the OGP markings is different from the ordinary product descriptions OGP descriptions are mostly "call to action" descriptions that are adjusted for users of social networks. Most of the web shops are using them in that way. Only 18 web shops use Microformats in comparison to Microdata that use 44 web shops, which are understandable, since it is the most recent recommendation of major search engines. Surprisingly, there is no use of any other vocabulary or ontology except

\footnotetext{
${ }^{6}$ http://www.alexa.com/topsites/category/Top/Shopping
} 
Schema.org. Also the namespaces that are used are reduced to three main groups: "product", "offer" and "aggreagateRating." More detailed use of other elements is missing. It is also surprising the fact that none of the web shops did use RDFa to indicate structural information about the products. RDFa is used only for OGP in the header. None of the studied web shops use GoodRelations ontology.

TABLE I: FoRMATS OF SEMANTIC ANNOTATION OF PRODUCTS USED By TOP 100 WEB SHOPS

\begin{tabular}{ll}
\hline \hline Format & Number of web shops \\
\hline RDFa (OGP) & 66 \\
Microdata & 44 \\
Microformats & 18 \\
\hline \hline
\end{tabular}

TABLE II: FORMATS AND NAMESPACES (VOCABULARY) USED BY TOP 100 WEB SHOPS

\begin{tabular}{lll}
\hline \multicolumn{1}{c}{ Format } & \multicolumn{1}{c}{ Namespace } & $\begin{array}{c}\text { Number of web } \\
\text { shops }\end{array}$ \\
\hline RDFa & opengraphprotocol.org/schema & 66 \\
\hline Microdata & schema.org/product & 40 \\
& schema.org/offer & 30 \\
& schema.org/AggregateRating & 23 \\
& schema.org/organization & 7 \\
& schema.org/review & 8 \\
& schema.org/rating & 7 \\
& schema.org/bredcrumb & 6 \\
& other & 10 \\
\hline Microformat & hreview-aggregate & 12 \\
& hproduct & 10 \\
& hreview & 2 \\
\hline \hline
\end{tabular}

Similar research on a larger corpus of data has shown an upward trend in the use of RDFa and Microdata, while stagnation of Microformats comparing the year 2010 and 2012 [10]. It is obvious that Schema.org since the year 2011 has greatly influenced the use of formats for labeling which led to these results, at least in the domain of e-commerce. We can conclude that era of Microformats slowly passes and Microdata markups are increasingly spreading.

Limitation of this research is reflected in the use of relatively small source of data (product pages of top 100 web shops) and no possibility of comparison with previous researches in the domain of e-commerce. Remains unexplored to what extent the formats for labeling of products were used in the corpuses from the 2009/10 and the year 2012 analyzed by [10]. It is not known to what extent are certain e-commerce ontologisms used in Linked data, all of which represent a challenge for future research.

\section{APPENDIX}

APPENDIX I: TOP 100 WEB SHOPS USED IN RESEARCH

\begin{tabular}{clrl} 
& APPENDIX I: TOP 100 WEB SHOPS USED IN RESEARCH \\
\hline \hline 1 & amazon.com & 51 & forever21.com \\
2 & ebay.com & 52 & wiley.com \\
3 & netflix.com & 53 & hsn.com \\
4 & amazon.co.uk & 54 & jcrew.com \\
5 & walmart.com & 55 & yoox.com \\
6 & Ikea.com & 56 & sephora.com
\end{tabular}

${ }^{7}$ http://www.google.com/webmasters/tools/richsnippets
7 bestbuy.com

8 target.com

9 groupon.com

10 homedepot.com

11 bodybuilding.com

12 newegg.com

13 lowes.com

14 macys.com

15 sears.com

16 gap.com

17 autos.yahoo.com

18 sky.com

19 nordstrom.com

20 zappos.com

21 dx.com

22 overstock.com

23 staples.com

24 barnesandnoble.com

25 bhphotovideo.com

26 nike.com

27 hm.com

28 kohls.com

29 ticketmaster.com

30 souq.com

31 costco.com

32 autotrader.com

33 shutterfly.com

34 legacy.com

35 livingsocial.com

36 tigerdirect.com

37 cars.com

38 walgreens.com

396 pm.com

40 jcpenney.com

41 cvs.com

42 directtv.com

43 sony.com

44 gamestop.com

45 victoriassecret.com

46 bedbathandbeyond.com

47 trademe.co.nz

48 cafepress.com

49 stubhub.com

50 samsclub.com
57 pixmania.com

58 cabelas.com

59 shopbop.com

60 officedepot.com

61 iherb.com

62 neimanmarcus.com

63 net-a-porter.com

64 rei.com

65 focalprice.com

66 marksandspencer.com

67 urbanoutfitters.com

68 cambridge.org

69 fineartamerica.com

70 redbubble.com

71 saksfifthavenue.com

72 weightwatchers.com

73 modcloth.com

74 abebooks.com

75 futureshop.ca

76 drugstore.com

77 bloomingdales.com

78 potterybarn.com

79 vitacost.com

80 mapsofindia

81 dickssportinggoods.com

82 musiciansfriend.com

83 uk.moo.com

84 frys.com

85 officemax.com

86 revolveclothing.com

87 play.com

88 scholastic.com

89 adorama.com

90 landsend.com

91 anthropologie.com

92 cduniverse.com

93 petco.com

94 micromaxinfo.com

95 harborfreight.com

96 ae.com

97 createandbarrel.com

98 dish.com

99 cargurus.com

100 dsw.com

\section{REFERENCES}

[1] B. J. Corbitt, T. Thanasankit, and H. Yi, "Trust and e-commerce: a study of consumer perceptions," Electronic Commerce Research and Applications, vol. 2, no. 3, pp. 203-215, 2003.

[2] D. Vandic, J. V Dam, and F. Frasnicar, "Facted product search powered by the semantic web," Decision Support Systems, vol. 53, pp. 425-437, June 2012.

[3] T. Berners-Lee, J. Hendler, and O. Lassila, "The semantic web," Scientific American, no. 284.5, pp. 28-37, 2001.

[4] M. M. Taye, "Understanding semantic web and ontologies: theory and applications," Journal of Computing, vol. 2, no. 6, pp. 182-192, June 2010.

[5] S. Pohorec, M. Zorman, and M. Kokol, "Analysis of approaches to structured data on the web," Computer Standards \& Interfaces, vol. 36, pp. 256-262, November 2013.

[6] R. Khare, "Microformats: the next (small) thing on the semantic web?" Internet Computing, IEEE, vol. 10, no. 1, pp. 68-75, 2006.

[7] J. Ronallo, "HTML5 microdata and schema.org," Code4Lib Journal, no.16, February 2012.

[8] B. Adida, M. Birbeck, S. McCarron, and S. Pemberton, RDFa in XHTML: Syntax and Processing, W3C Recommendation, 2008.

[9] M. Hepp, "GoodRelations: an ontology for describing products and services offers on the web," in Proc. the 16th International Conference 
on Knowledge Engineering: Practice and Patterns, Acitrezza, 2008 , vol. 5268, pp. 329-346.

[10] H. Mühleisen and C. Bizer, "Web data commons - extracting structured data from two large web corpora," in Proc. CEUR Workshop, Lyon, 2012, vol. 937.

[11] P. Mika and T. Potter, "Metadata statistics for a large web corpus," in Proc. CEUR Workshop, Lyon, 2012, vol. 937.

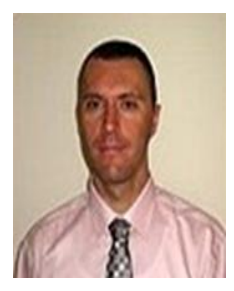

Goran Matošević was born in 1977 in Pula, Croatia. He graduated from the Faculty of Economics in Rijeka in the year of 2003 and he finished his master's degree at the Faculty of Economics in Zagreb in the year of 2009, the direction of IT management Currently he is enrolled in a doctoral program in information science at the Faculty of Organization and Informatics in Varaždin.

He works at the Faculty of Economics and Tourism, at the University of Pula as a research assistant. Previously, he was employed as a web developer in several ICT companies. His areas of interests include the semantic web, information retrieval, web technologies and e-commerce. 